\title{
THE STRUCTURE OF THE CHROMOSPHERE AND THE LOW CORONA
}

\author{
C. DE JAGER \\ Sonnenborgh Observatory, Utrecht, The Netherlands
}

The outer part of the sun consists of two main regions: (1) the convection zone, which extends from a depth of $10^{5} \mathrm{~km}$ below the visible surface to 100 or $200 \mathrm{~km}$ below the surface, and in which the temperature decreases from about $10^{\circ}{ }^{\circ} \mathrm{K}$ near the lower limit to $6000^{\circ} \mathrm{K}$ near the surface; and (2) the corona, which starts about $5000 \mathrm{~km}$ above the surface and extends very far, up to many solar radii, and in which the temperature is $10^{\circ}{ }^{\circ} \mathrm{K}$ in the low parts.

Between these there is a very thin transition region. The lowest few hundred kilometers of it, which are still opaque for visual light, are called the photosphere; the rest is called the chromosphere.

The chromosphere is, therefore, a transition between the relatively cool photosphere and the hot corona. The dynamical features observed in the chromosphere are largely caused by the dissipation of mechanical energy, which goes from the upper part of the convection zone through the upper photosphere and chromosphere into the corona.

When seen at the limb, the chromosphere shows a spiky structure; these spicules are visible only above $5000 \mathrm{~km}$ and emit principally a line spectrum. Their average diameter is of the order of $1000 \mathrm{~km}$; their lifetimes are of the order of some minutes. In the lower chromospheric levels $(<5000 \mathrm{~km})$ the inhomogeneities can be seen from above on spectroheliograms (monochromatic pictures of the sun). We distinguish between:

\begin{tabular}{|c|c|c|c|}
\hline Fine mottles & $\begin{array}{c}\text { Diameter } \\
10^{3} \mathrm{~km}\end{array}$ & $\begin{array}{l}\text { Lifetime } \\
5^{\mathrm{m}}\end{array}$ & Remarks \\
\hline Coarse mottles & 5 to $8 \times 10^{3} \mathrm{~km}$ & one day? & $\begin{array}{l}\text { cluster of } \\
\text { fine mottles }\end{array}$ \\
\hline $\begin{array}{l}\text { Chromospheric } \\
\text { network }\end{array}$ & $50,000 \mathrm{~km}$ & days? & $\begin{array}{l}\text { composed of } \\
\text { coarse mottles }\end{array}$ \\
\hline
\end{tabular}

It seems that the fine mottles may be identified with the spicules seen from above.

In any case, such observations make it clear that in representing the atmospheric structure by a set of numbers, i.e. in constructing a chromospheric model, one must distinguish at least between the hot and cold (dense and tenuous) parts of the chromosphere. Several such models have been constructed in the last few years. 


\section{CHROMOSPHERIC MODELS}

Fig. 1 summarizes recent chromospheric models. A critical examination shows that the great differences between them are less important and that an average model can be constructed that roughly tallies with the observational data:

1. All models show a sharp increase in temperature between 4000 and $10,000 \mathrm{~km}$; the position of this increase, as determined from radio observations, is indefinite, but according to optical eclipse observations (see also the model AMT) this increase should occur close to $4000 \mathrm{~km}$.

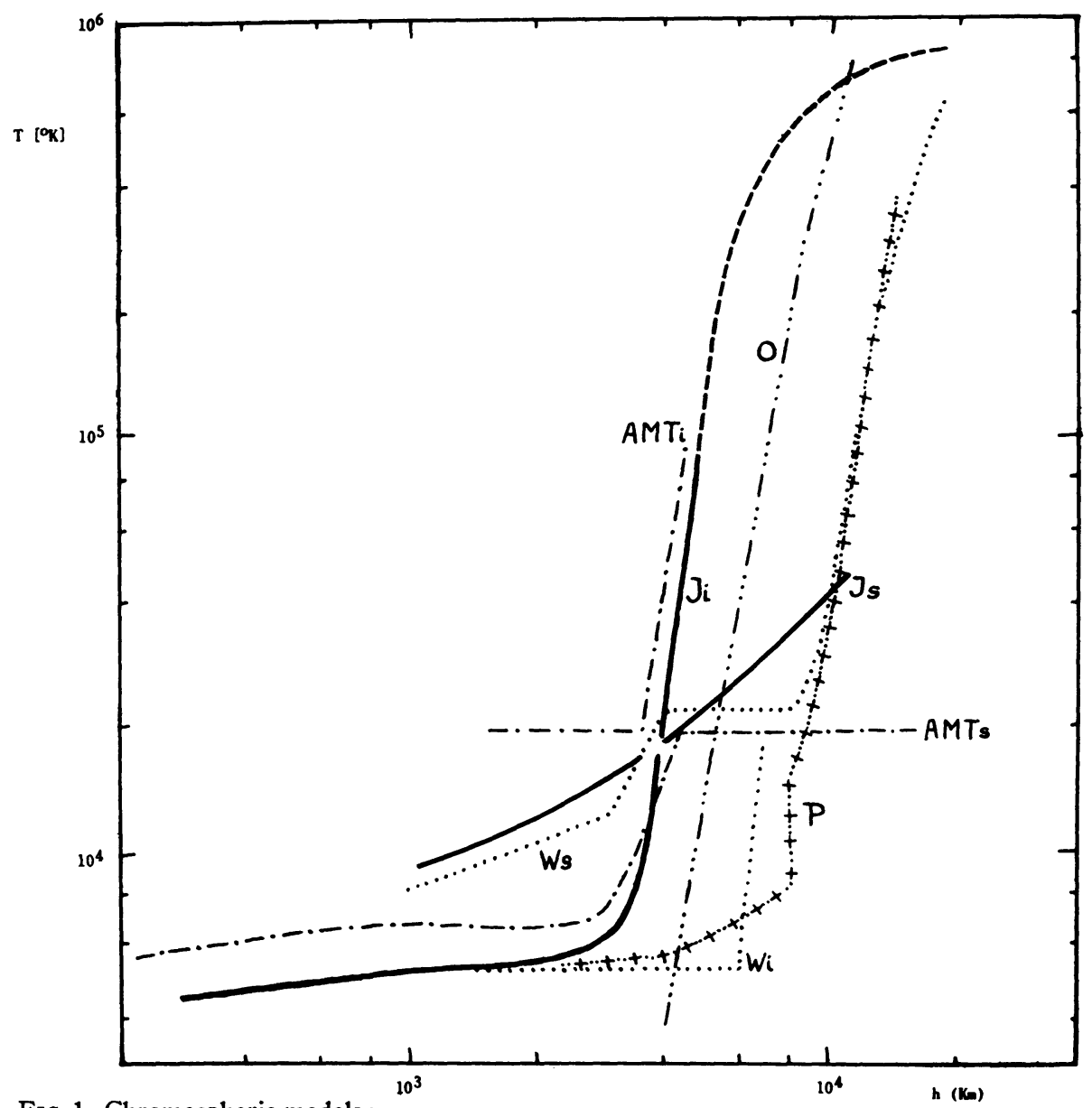

FIG. 1. Chromospheric models :

$A M T$-R. G. Athay, D. H. Menzel, and R. N. Thomas, Ap. J. 123, 285, 1955 and I.A.U. Colloquium 4, 279, 1957.

$O$-L. Oster, Z. Ap. 40, 28, 1956.

$P$-J. H. Piddington, Ap. J. 119, 531, 1954.

W-L. Woltjer, B.A.N. 12, 165, 1954.

J-C. de Jager, Handbuch der Physik, 52, 141, 1959.

$s p$-spicules; $i$-interspicular matter. 
2. Below $4000 \mathrm{~km}$ the main part of the chromosphere consists of cool elements with temperatures between 4000 and 6000 degrees.

3. There is some controversy on the temperature of the hot elements below $4000 \mathrm{~km}$, but it seems that $T_{e}$ rises from about $10^{4}{ }^{\circ} \mathrm{K}$ near $1000 \mathrm{~km}$ to $2 \times 10^{4}{ }^{\circ} \mathrm{K}$ near $4000 \mathrm{~km}$.

4. Recent spectral spicule observations $[1,2]$ indicate that above $5000 \mathrm{~km}$, where the spicules are seen penetrating into the corona, their temperature is of the order of 3 to $6 \times 10^{4}{ }^{\circ} \mathrm{K}$ near $10,000 \mathrm{~km}$.

These considerations have led to the construction of an average model ( $J_{i}$ or $J_{s p}$ in Fig. 1). It should be remarked that it is not yet quite certain whether the hot or the cold elements of the low chromosphere are the bases of the spicules; discussions of spectroheliographic structures suggest that it is the hot ones.

\section{ENERGY BALANCE OF THE CHROMOSPHERE}

In Table I we give the energy balance of the chromosphere for some characteristic heights. (The table gives the logarithm of the energy flux in ergs $/ \mathrm{cm}^{2} /$ second.)

\section{TABLE I}

\begin{tabular}{|c|c|c|c|c|}
\hline & $\begin{array}{l}\text { otospheric } \\
\text { diation Flux }\end{array}$ & $\begin{array}{l}\text { Mechanical } \\
\text { Flux }\end{array}$ & $\begin{array}{c}\text { Emission of } \\
\text { Radiation }\end{array}$ & $\begin{array}{l}\text { Conductive } \\
\text { Flux }\end{array}$ \\
\hline Chromosphere $\left\{\begin{array}{r}100 \mathrm{~km} \\
4000 \mathrm{~km}\end{array}\right.$ & $\begin{array}{l}10.8 \\
10.8\end{array}$ & $\begin{array}{l}8.7 \\
5.9\end{array}$ & $\begin{array}{l}8.7 \\
6.3\end{array}$ & \\
\hline Transition Layer & 10.8 & & 5.4 & 5.6 \\
\hline Corona & 10.8 & & 4.5 & \\
\hline
\end{tabular}

\section{EXPLANATION}

The mechanical energy flux is the irrotational part of the photospheric turbulence spectrum, progressing outward and partly losing its energy to heat the chromosphere (perhaps thereby producing the fine mottling and the spicules). The mechanical flux is computed as $F_{m}=\frac{1}{2} \rho V_{t}^{2} c$, where $\rho$ is the density, $V_{t}$ is the vertical turbulent velocity component, and $c$ is the velocity of sound. We have [3]:

$\begin{array}{lcc}h(\mathrm{~km}) & 100 & 4000 \\ V_{t}(\mathrm{~km} / \mathrm{sec}) & 3 & 15 \\ \log N & 15.7 & 11.9 \\ \log F_{m} & 8.7 & 5.9\end{array}$

Clearly $F_{m}$ decreases outward so that an important part of the mechanical flux appears to be used to heat the low chromosphere. Schematically, this situation may be illustrated as in Fig. 2(a): in a volume element with a height equal to the scale height $H$, about half of the incoming mechanical energy flux $\left(F_{m}\right)_{\text {in }}$ is dissipated and finally emitted as radiation $\left(F_{\mathrm{rad}}\right)$; the other half 

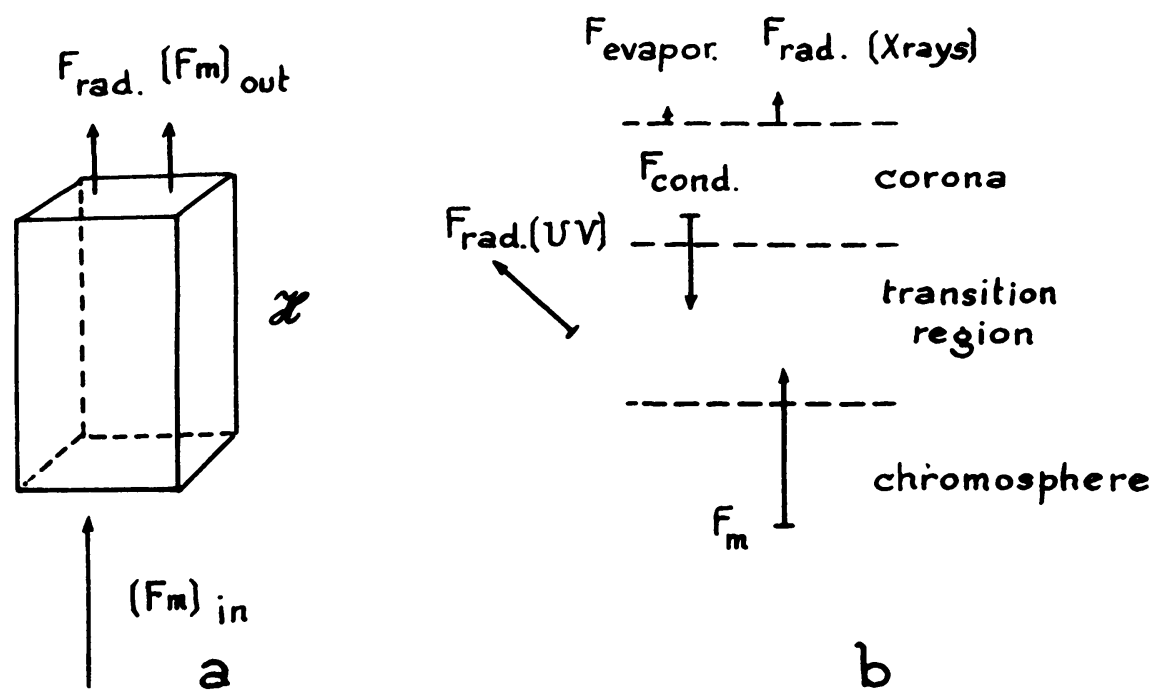

Fig. 2. Energy balance in the chromosphere: $(a)$ for a volume element with a height equal to the scale height $H$ at the $4000-\mathrm{km}$ level (probably representative for the mean chromosphere); $(b)$ in the transition region. The situation has been idealized in the sense that the energy balance, indicated by the length of the arrow, is perfect, whereas this is not wholly the case for the data given in the above table. Thus in Fig $1(a):\left(F_{m}\right)_{\text {In }}=\left(F_{m}\right)_{\text {out }}+F_{\text {rad }}$; and in Fig. $2(b): F_{m}=F_{\text {cond }}+F_{\mathrm{rad}(x)}+F_{\text {evap, }}$ and $F_{\text {cond }}=F_{\mathrm{rad}(u r)}$.

is not dissipated and progresses outward, $\left(F_{m}\right)_{\text {out }}$. This situation may be representative for the main chromosphere.

In the transition region to the corona (Fig. $2(b)$ ), the situation is somewhat more complicated. Because of the density decrease above $4000 \mathrm{~km}$, the emission of radiation of a unit-volume element becomes considerably lower than the dissipation of energy $\left(\approx 10^{6} / H\right)$ per unit of volume. Hence, in a height range of about the scale height $\approx 10^{3} \mathrm{~km}$ a sharp increase in temperature must occur, which explains the sudden rise in temperature from chromospheric to coronal values.

What happens further to the mechanical energy flux that heated the corona is shown in Fig. $2(b)$.

1. A small part of it is lost by radiation, mainly in the $X$-ray region.

2. A presumably still smaller part is lost by evaporation: the coronal kinetic temperatures are so high that there is a constant evaporation of highvelocity particles.

3. The greater part of this energy is conducted backward toward the chromosphere (the corona and chromosphere are good heat conductors). This smoothes the temperature gradient in the transition layer, so that the temperature distribution in this layer is described mainly by the conduction equation [4]:

$$
\pi F_{c}=K d T / d h,
$$


where the conductive flux $\pi F_{\mathrm{c}}$ at the top of the transition layer can be determined from the observational values of $d T / d h$ and from the theoretical ones of $K\left(T, N_{e}\right)$ [5].

The flux computed from the observational $T(h)$ curve (see next section) appears to be of the same order as the incoming mechanical flux, and this conductive flux, in turn, is finally radiated as the emission lines of the far ultraviolet solar spectrum (near $1000 \AA$ ), which are emitted by regions with temperatures between $10^{4}$ and $3 \times 10^{5}{ }^{\circ} \mathrm{K}$, hence, by the transition layer.

This closes the energy balance of the transition region.

\section{THE TRANSITION REGION}

The transition region between the chromosphere and the corona is one of most inaccessible parts of the solar atmosphere. The conditions prevailing in it can be studied by making use of $(a)$ the rocket ultraviolet emission spectrum, but this study is hampered by the lack of knowledge of transition probabilities and of accurate photometric data; $(b)$ the detailed radio spectrum of the quiet sun as discussed recently by Allen [6,3]; and $(c)$ the detailed data on brightness distribution of the quiet minimum sun, since these are now known for polar and equatorial radii from different kinds of observations (eclipse, interferometer, pencil beam) made in the years around 1954 (see Fig. 3).

This material has been submitted to investigation at Utrecht to determine the temperature variation in the transition region. At the moment of writing, this investigation was not yet finished, but the following preliminary $T(h)$ function has been derived:

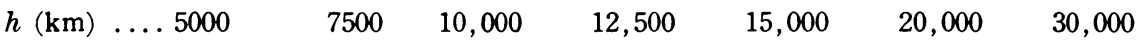

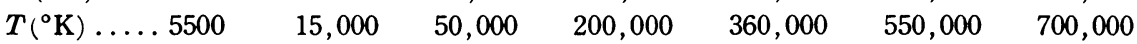

This result is still subject to corrections; the $h$-values, for instance, could still be increased or decreased by a constant amount. The function, however, is precise enough to determine $\pi F_{c}$ (see Table I) with sufficient accuracy and a shift in $h$ would not influence this quantity. In this discussion, two approximations have been applied:

1. The contribution of hot elements of the low chromosphere and of the spicules has been neglected. The radio spectrum of the quiet sun at $\lambda \leq 1 \mathrm{~cm}$ shows that the chromosphere below $5000 \mathrm{~km}$ consists chiefly of a cool gas with $T_{e}<6000$ degrees. The relative volume occupied by hot elements must be small, at least smaller than 30 per cent. Since the number of spicules above $5000 \mathrm{~km}$ is certainly small (of the order of some per cent only), the solar radio spectrum informs us primarily on the interspicular matter (above $5000 \mathrm{~km}$ ) and on the cool parts of the low chromosphere.

2. It is known [7] that at long meter wavelengths the brightness distribution over the disk is partly determined by inhomogeneities in the upper corona. We have the impression that these inhomogeneities do not play a part in the intensity distribution in decimeter waves. 

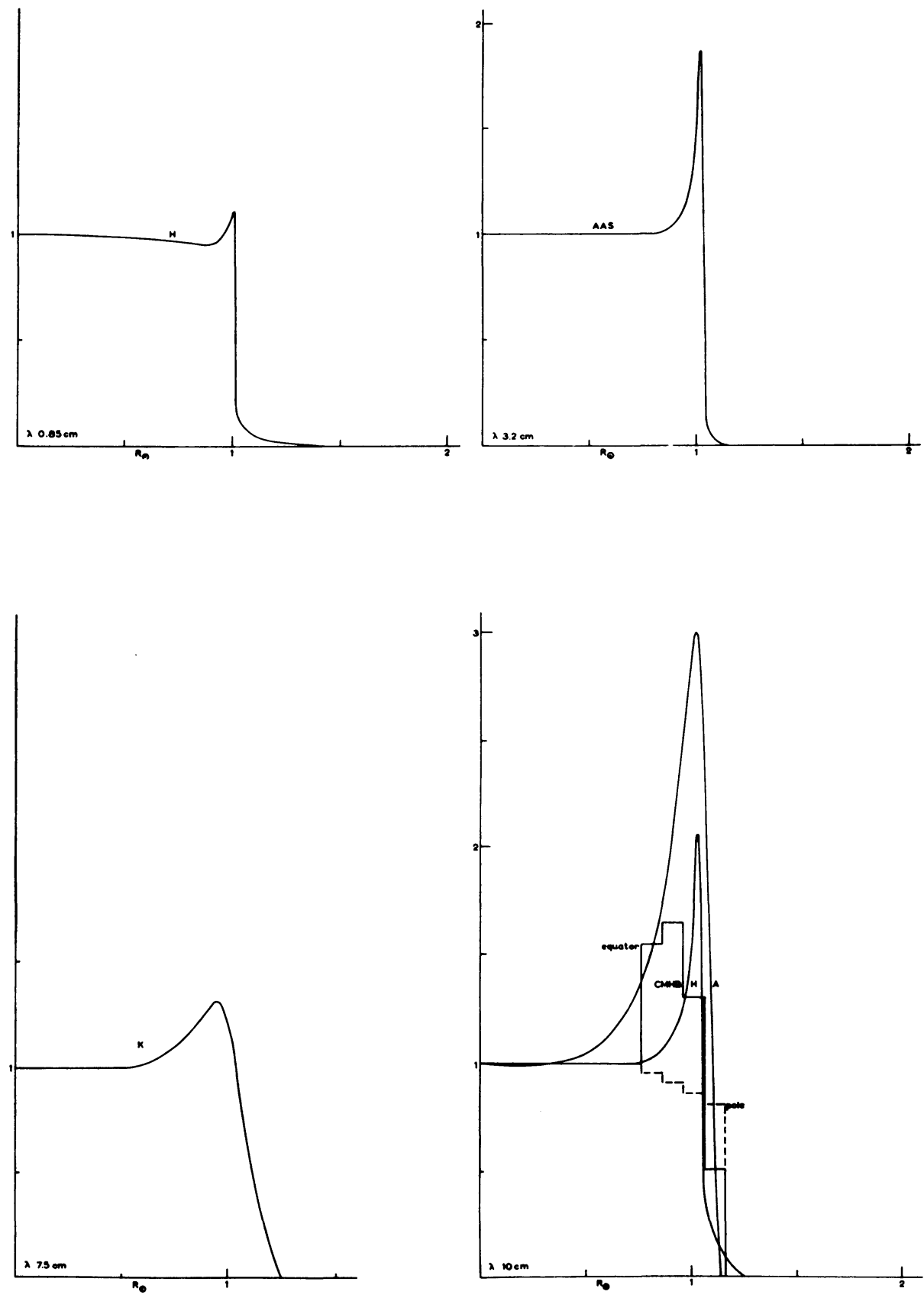

FIG. 3. Center-limb variation of the brightness temperature of the quiet sun. 

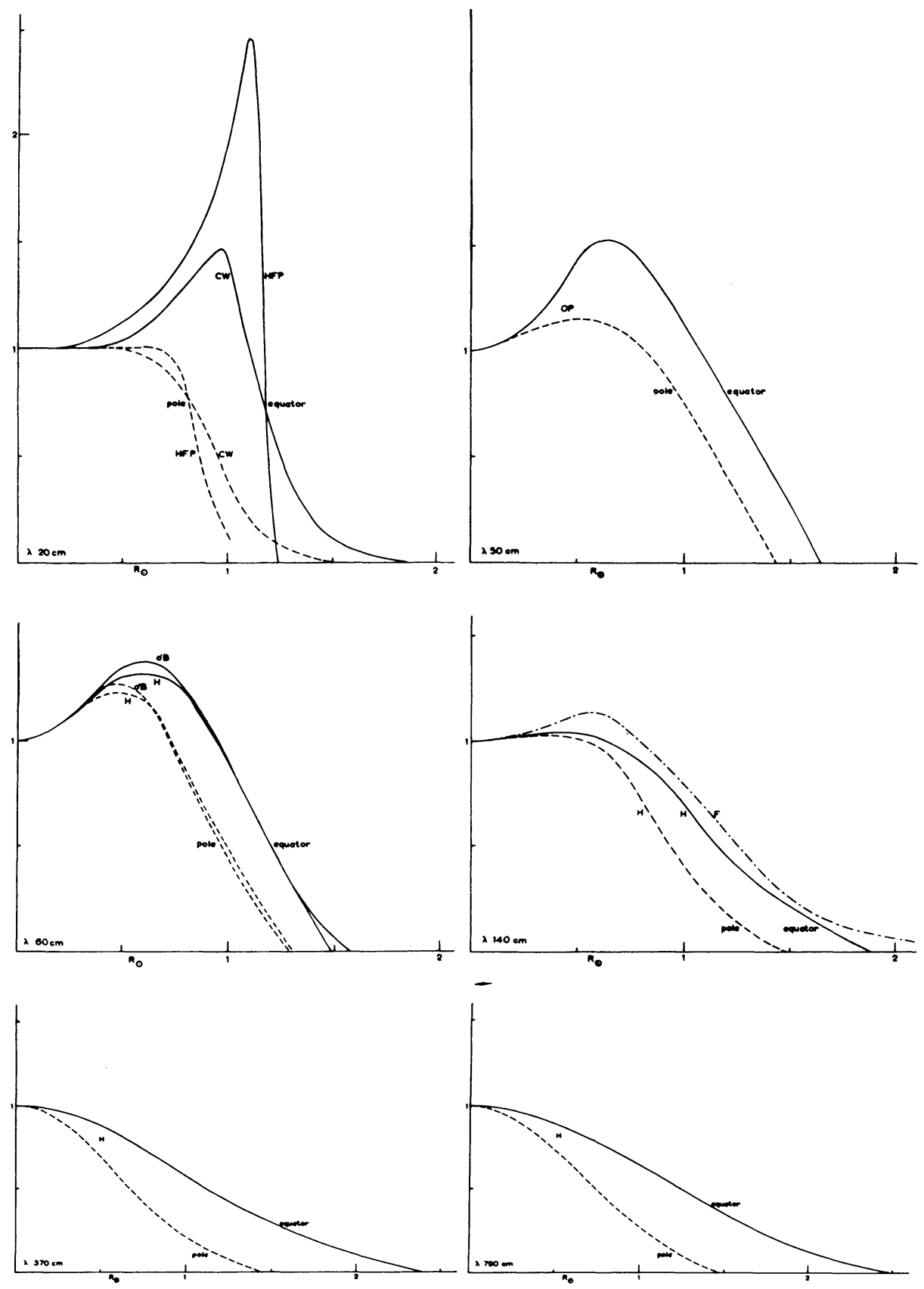

FIg. 3. Center-limb variation of the brightness temperature of the quiet sun. 


\section{THE 3.03-CM LINE}

A possible further source of information on the region between the chromosphere and the corona could be the line at $3.03 \mathrm{~cm}$, originating from the $2^{2} S_{1 / 2} \rightleftarrows 2^{2} P_{3 / 2}$ transition of neutral hydrogen [8]. This line's transition probability is $2 \times 10^{8}$ times greater than that of the $21-\mathrm{cm}$ line.

Since the collisional transition probability between the $2 P$ and $2 S$ levels is great, their relative populations will assume thermodynamic equilibrium, and the appearance of the line (emission or absorption) will depend on the temperature gradient in the chromospheric levels, in which the optical depth $\tau_{\lambda}$ at $3.03 \mathrm{~cm}$ is of order unity; the value $\Delta T$; the excess of the line's brightness temperature over that of the adjacent continuous $T_{b}$ ) will be of the order

$$
\frac{\kappa_{\text {line }}}{\kappa_{\text {cont }}} \times \frac{d T}{d \tau_{\lambda}}
$$

where $\kappa$ is the absorption coefficient.

Supposing that the hydrogen levels are populated as in thermodynamic equilibrium (which is not the case!), one finds the following values for $\log \left(\kappa_{\text {cont }} / \kappa_{\text {line }}\right)$ :

$\begin{array}{rccccccc}\log N_{e} \quad T\left({ }^{\circ} \mathrm{K}\right) 5000 & 7500 & 10,000 & 15,000 & 20,000 & 25,000 & 40,000 \\ 8 \ldots \ldots \ldots & 5.4 & 6.6 & 7 \cdot 1 & 5.9 & 5.0 & 4.5 & 3.8 \\ 9 \ldots \ldots & 5.4 & 6.6 & 7.1 & 6.3 & 5.5 & 5.0 & 4.3 \\ 10 \ldots \ldots & 5.4 & 6.6 & 7.2 & 6.8 & 6.0 & 5.5 & 4.8 \\ 11 \ldots \ldots . & 5.4 & 6.6 & 7.2 & 7.1 & 6.5 & 6.0 & 5.3 \\ 12 \ldots \ldots \ldots & 5.4 & 6.6 & 7.2 & 7.6 & 7.0 & 6.5 & 5.8\end{array}$

The radiation at $3 \mathrm{~cm}$ is mainly emitted at $h=7000 \mathrm{~km}$, where $T=15,000^{\circ} \mathrm{K}$, $\log N_{e}=9$, and $d T / d \tau=10^{4}{ }^{\circ} \mathrm{K}$, yielding $\Delta T=0.005^{\circ} \mathrm{K}$. In that case the line would be invisible. These computations, however, have been made for local thermodynamic equilibrium conditions. It is known that in the chromosphere the second hydrogen level is considerably overpopulated, and in the literature we find overpopulation ratios, up to a factor $10^{3}$. In the latter case the line could be visible. Observations made at the Nera Observatory, Holland, did not yield a decisive result.

There would perhaps be an increased visibility of the line in $(a)$ prominence regions, since the temperature gradient therein may be steeper than in the quiet chromosphere (the inhibition of conduction by the magnetic field around prominences will steepen the temperature distribution); (b) active centers, where the increased density will put the level $\tau=1$ at higher temperatures than the increasing $\log \left(\kappa_{11 n e} / \kappa_{\text {cont }}\right)$.

For effective help in the greater part of the computations I owe many thanks to Mr. J. Repelaer van Driel.

\section{REFERENCES}

[1] Athay, R. G., and Thomas, R. N. Ap. J. 125, 804, 1957.

[2] Smith, E. v. P. Ap. J. 126, 529, 1957.

[3] de Jager, C. Handbuch der Physik, Vol. 52. Heidelberg (Springer), 1959, p. 81. 
[4] Unsöld, A. Physik der Sternatmosphären (2. Aufl.). Heidelberg (Springer), p. 2.

[5] Oster, L. Z. Ap. 42, 228, 1957.

[6] Allen, C. W. M.N.R.A.S. 117, 174, 1957.

[7] Scheffler, H. Z. Ap. 45, 113, 1958.

[8] Wild, J. P. Ap. J. 115, 206, 1952.

\section{Discussion}

Thomas: In calculating the radiative emission in the chromosphere, which did you find most important, lines or free-bound continuous emission?

de Jager: Lines.

Thomas: Which lines, Balmer or Lyman? What did you do about the transfer problem? Or did you just consider the emission from a thin atmosphere, and no transfer problem?

de Jager: I just used the observed lines and observed emission. The values given for the radiation of the chromosphere are the observed emission intensities in the visual region, multiplied by an arbitrary factor 2 for the contribution of the ultraviolet and infrared spectral regions. Naturally, these values only represent orders of magnitude.

Takakura: Is the temperature $\left(\Delta T=0.005^{\circ} \mathrm{K}\right)$ of the line emission at 3.03 $\mathrm{cm}$ the apparent temperature?

de Jager: It is predicted that the $3.03-\mathrm{cm}$ line will appear as an emission line with a brightness temperature exceeding that of the neighboring continuum by $0.005^{\circ} \mathrm{K}$. 